\title{
Type II conventional dendritic cells of asthmatic patients with frequent exacerbations have an altered phenotype and frequency
}

To the Editor:

In recent decades it has become clear that dendritic cells (DCs) critically contribute to the development of $\mathrm{T}$ helper 2 (Th2)-mediated allergic diseases, such as asthma. Two main conventional DC (cDC) subsets, type $1 \mathrm{cDCs}(\mathrm{cDC} 1 \mathrm{~s})$ and $\mathrm{cDC} 2 \mathrm{~s}$, can be identified based on the expression of cell-surface molecules and transcription factors [1]. Using mouse models in which specific DC subsets are targeted or by adoptive transfer of DC subsets, it has been shown that upon activation in the airways cDC2s migrate towards the lung-draining lymph node where they induce allergen-specific Th2 cells, which subsequently promote eosinophilic airway inflammation [2]. The role of $\mathrm{cDC} 1 \mathrm{~s}$ in asthma is more controversial, although most recent reports indicate that $\mathrm{cDC} 1 \mathrm{~s}$ can efficiently suppress allergic airway inflammation [3], either by the induction of regulatory T-cells [4] or through increased interleukin (IL)-12 production [5]. These functional properties of DC subsets were evaluated in mouse models, but to date it is unclear how these findings reflect the characteristics of cDC subsets in asthmatic patients with different disease severities. Therefore, we questioned whether DC subset frequencies and the surface expression of co-stimulatory and co-inhibitory molecules differ between healthy persons and asthma patients. To investigate this, we focused on the Th2-stimulatory molecules (CD86, OX40L) and Th2-inhibitory molecule PD-L1 [6-8].

We used multicolour flow cytometry to identify DC subsets and their expression levels of co-stimulatory and co-inhibitory markers in peripheral blood [9]. We characterised cDCs in 59 asthma patients that were treated with inhaled corticosteroids corresponding with a Global Initiative for Asthma 3/4 score and 28 age- and sex-matched healthy individuals. The study was approved by the medical ethical committee of the Erasmus MC Rotterdam, and written informed consent was obtained from every participant prior to study inclusion. Patients were divided into three subgroups, based on the number of exacerbations in the year of blood withdrawal $(0,1$ and $>1)$. The proportion of eosinophils was increased in asthma patients as compared to healthy individuals, but was independent of the number of exacerbations per year (data not shown). After exclusion of T-cells, B-cells, and CD14 or CD16-expressing monocytes, total cDCs are characterised based on expression of CD11c and low expression of CD123, herein CDC1s and cDC2s were characterised based on intracellular expression of IRF8 or IRF4, respectively (figure 1a). The frequency of total cDCs in peripheral blood mononuclear cells was reduced in asthma patients compared with healthy controls (figure $1 \mathrm{~b}$ ). This decrease was most pronounced in asthmatic patients that exhibit more than one exacerbation per year. Within cDCs, a specific decrease in the proportion of cDC1s was observed, whereas the relative proportion of $\mathrm{cDC} 2 \mathrm{~s}$ was increased in asthma patients. Similar results were observed when a ratio was determined between $\mathrm{cDC} 2 \mathrm{~s}$ and $\mathrm{cDC} 1 \mathrm{~s}$ (data not shown). Strikingly, the increase in $\mathrm{cDC} 2$ was particularly observed in patients with multiple exacerbations per year (figure 1b).

Conventional DC2s of asthma patients expressed lower levels of PD-L1 compared with healthy individuals, particularly cDC2s from patients with more than one exacerbation per year (figure 1d). Also, the expression levels of costimulatory molecules CD86 and OX40L in cDC2s of asthma patients were

@ERSpublications

Proportions of conventional dendritic cell subsets and the expression of co-stimulatory and coinhibitory molecules in $\mathrm{cDC} 2 \mathrm{~s}$ are altered in asthma patients. These changes are most pronounced in asthmatics with frequent exacerbations. http://bit.ly/2wbb8UJ

Cite this article as: Vroman H, Tindemans I, Lukkes M, et al. Type II conventional dendritic cells of asthmatic patients with frequent exacerbations have an altered phenotype and frequency. Eur Respir J 2020; 55: 1900859 [https://doi.org/10.1183/13993003.00859-2019]. 

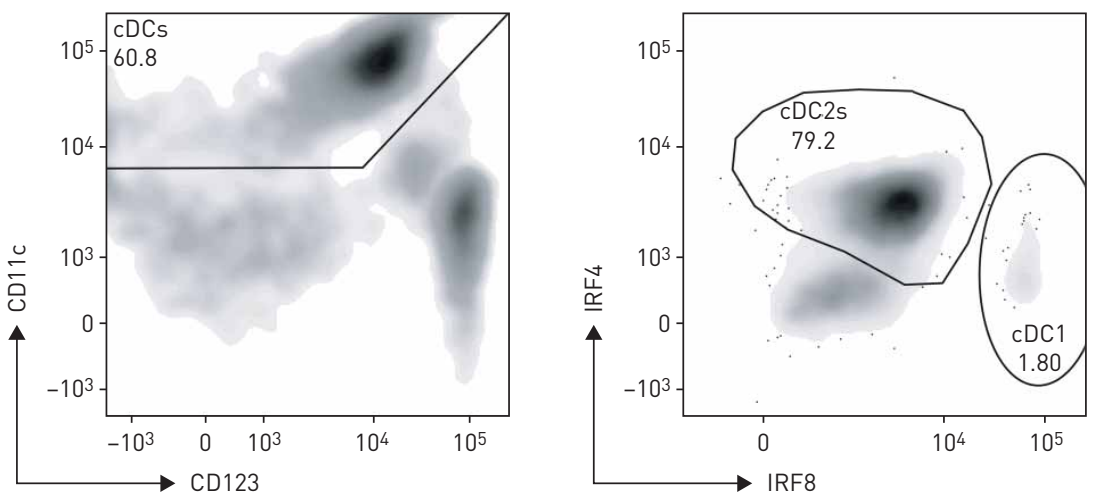

b)

Total cDCs
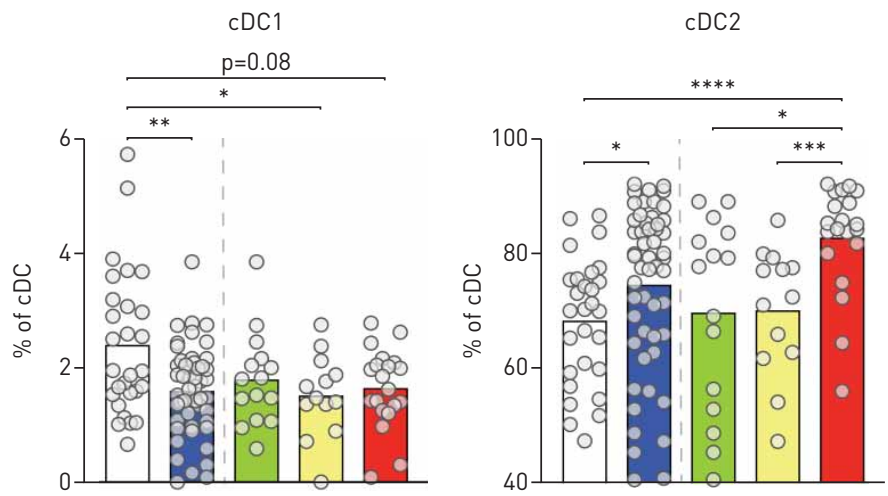

c)

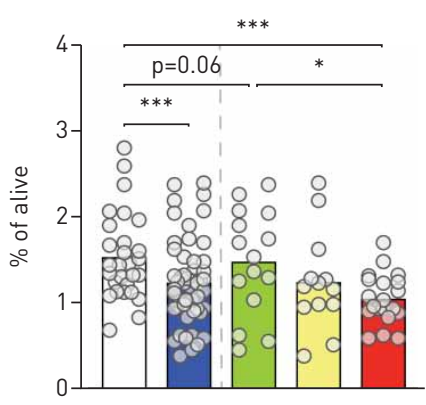

$\mathrm{cDC} 2$

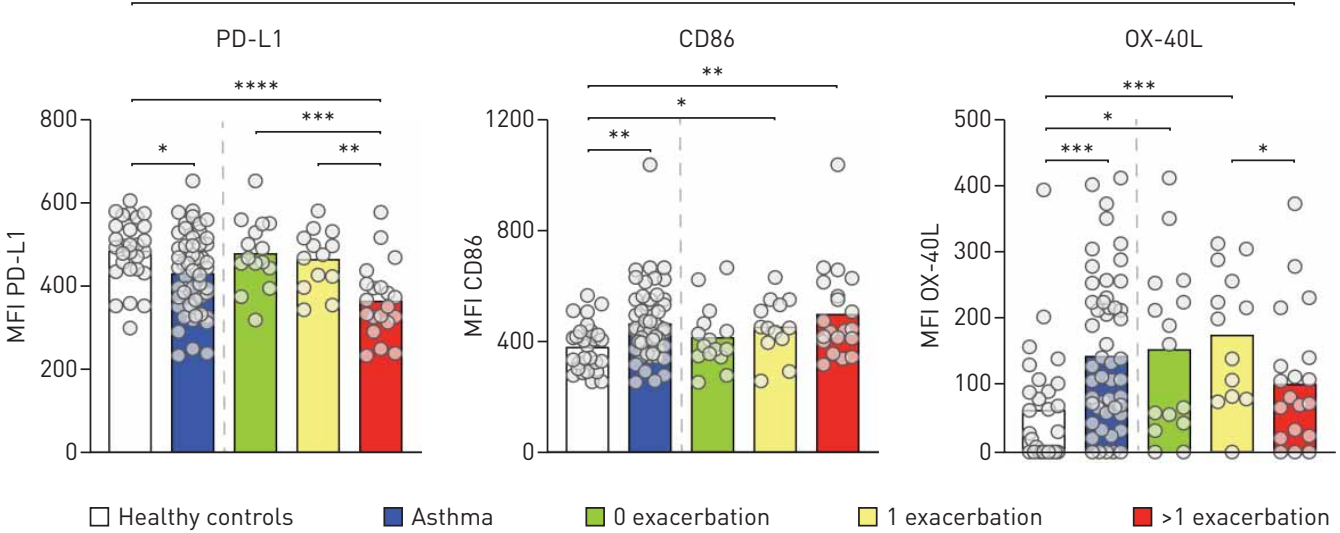

FIGURE 1 Altered expression of PD-L1, HLA-DR, CD86 and OX4OL in conventional dendritic cells (cDCs) of asthmatic patients. a) Gating strategy for cDC1s and cDC2s in PBMCs by flow cytometry. b) Frequency of total $\mathrm{cDCs}, \mathrm{cDC} 1 \mathrm{~s}$ and $\mathrm{cDC} 2 \mathrm{~s}$ in peripheral blood mononuclear cells (PBMCs) of healthy controls and asthma patients by flow cytometry. c) Enumeration of the MFI of PD-L1, CD86, and OX-40L on cDC2s of healthy controls and asthma patients by flow cytometry. The following flow cytometric antibodies were used to characterise dendritic cell subsets and expression of co-stimulatory and co-inhibitory molecules; CD11c (clone 3.9), CD14 (clone M5E2), CD16 (clone 3G8), CD86 (clone 2331 (FUN-1)), CD123 (clone 7G3), IRF4 (clone 3E4), IRF8 (clone V3GYWCH), PD-L1 (clone M1H1), HLA-DR (clone G46-6) and OX-40L clone ik-1). The lineage mix contained CD3 (clone UCHT1), CD19 (clone HIB19) and CD20 (clone 2H7). IRF4 and IRF8 staining was performed intra-cellularly, as described previously [9]. Data was acquired on a LSRII (BD Biosciences). Significance was determined using a Mann-Whitney U-test. *: $p<0.05 ;{ }^{* *}$ : $p<0.01{ }^{* * *}$ : $p<0.001$.

increased compared to controls (figure 1d). OX-40L expression was highest on cDC2s from patients with no or one exacerbation in the year of blood withdrawal.

Frequencies of cDCs and expression of co-inhibitory and co-stimulatory markers on cDC2s did not correlate with other clinical parameters such as fractional exhaled nitric oxide, lung function (forced 
expiratory volume in $1 \mathrm{~s}$ ), IgE levels, or eosinophil count and proportions in peripheral blood (data not shown). Although the Asthma Control Questionnaire score significantly correlated with the number of exacerbations per year, it was not predictive for $\mathrm{CDC}$ frequencies or phenotype (data not shown).

Alterations in the proportion of cDC subsets and their expression of co-stimulatory and co-inhibitory molecules could contribute to the predisposition of asthmatics to develop Th2-associated inflammation, as is already shown in murine experimental asthma models [6-8].

In line with these murine experiments, we found decreased expression of the co-inhibitory molecule PD-L1 expression on $\mathrm{CDC} 2 \mathrm{~s}$ of asthma patients with frequent exacerbations and not in patients without exacerbations. Reduced PD-L1 expression is associated with disease severity, as the expression during stable disease and exacerbation is not different (data not shown). Increased CD86 and OX-40L expression on DCs is crucial during the induction of Th2 responses in several experimental airway inflammation mouse models [6-8]. Interestingly, we have observed in our cohort that CD86 and OX-40L expression was increased in $\mathrm{cDC} 2 \mathrm{~s}$. These data suggest that $\mathrm{cDC} 2 \mathrm{~s}$ of asthmatics display a phenotype associated with Th2-inducing capacities. Unexpectedly, OX-40L expression on CDC2 was only elevated in patients with no or one exacerbation; however, this was not associated with a more pronounced Th2 inflammatory profile, as determined by eosinophil frequency and IgE levels. Additionally, lower OX-40L expression on cDC2s of asthma patients with multiple exacerbations might indicate involvement of other, Th2-independent, inflammatory processes, as has been suggested before [10].

Previously, no differences were observed in the absolute counts of cDCs between allergic patients and healthy individuals [11]. Additionally, increased numbers of DCs, expressing CD141, a marker specific for $\mathrm{cDC} 1 \mathrm{~s}$, were found, whereas $\mathrm{CD} 1 \mathrm{a}^{+} \mathrm{DCs}$, most likely representing $\mathrm{cDC} 2 \mathrm{~s}$, were decreased [11]. Similarly, increased frequencies of $\mathrm{CD} 141^{+}$DCs were found in HDM-atopic adolescents with asthma as compared to non-atopic individuals [12]. The differences between these studies and our findings could originate from 1) the analysis of cell frequencies instead of absolute cell counts, or 2) from the markers used to define the different DC subsets, and 3) patient characteristics. In our study, we relied on the transcription factors IRF4 and IRF8, which were shown to represent robust markers to distinguish cDC1s from cDC2s in many tissues and species [9]. Future research should show whether the observed changes in peripheral blood CDC frequencies and characteristics (as determined by IRF4 and IRF8 expression) correlate with the changes in the airways of asthma patients.

In summary, we have provided evidence that proportions of $\mathrm{cDC} 1 \mathrm{~s}$ and $\mathrm{cDC} 2 \mathrm{~s}$ are altered in peripheral blood of asthma patients, in favour of cDC2s, especially in patients with frequent exacerbations. In addition, the expression of OX-40L was specifically increased on cDC2s of asthma patients with none or few exacerbations, whereas cDC2s of asthma patients with frequent exacerbations expressed lower amounts of PD-L1. This suggests that the frequency and phenotype of $\mathrm{cDC} 2 \mathrm{~s}$ could differentiate between patients that exhibit a low or a high number of exacerbations per year, and should be studied in more detail in future research.

Heleen Vroman ${ }^{1}$, Irma Tindemans ${ }^{1}$, Melanie Lukkes ${ }^{1}$, Menno van Nimwegen ${ }^{1}$, Geertje M. de Boer $\odot^{2}$, Gerdien A. Tramper-Stranders $\oplus^{3}$, Gert-Jan Braunstahl $\oplus^{1,2}$, Rudi W. Hendriks ${ }^{1}$ and Mirjam Kool ${ }^{1}$

${ }^{1}$ Dept of Pulmonary Medicine, Erasmus MC, Rotterdam, The Netherlands. ${ }^{2}$ Dept of Respiratory Medicine, Franciscus Gasthuis and Vlietland, Rotterdam, The Netherlands. ${ }^{3}$ Dept of Pediatric Medicine, Franciscus Gasthuis and Vlietland, Rotterdam, The Netherlands.

Correspondence: Mirjam Kool, Erasmus MC, Dept of Pulmonary Medicine, Wytemaweg 80, Room Ee22-24a, Rotterdam 3015 CN, The Netherlands. E-mail: m.kool@erasmusmc.nl

Received: 26 April 2019 | Accepted after revision: 4 Feb 2020

Acknowledgements: We would like to thank all the participants of the study, and the physicians and research nurses of the Franciscus Gasthuis (Rotterdam, the Netherlands) for their assistance in study design and inclusion of participants. Additionally, we would like to thank B.W.S. Li, E. van der Ploeg, and M.J.W. de Bruijn for their assistance during the project.

Conflict of interest: H. Vroman reports grants from EAACI Young Investigator's Grant, during the conduct of the study. I. Tindemans has nothing to disclose. M. Lukkes has nothing to disclose. M. van Nimwegen has nothing to disclose. G.M. de Boer has nothing to disclose. G.A. Tramper-Stranders reports grants from OM Pharma, ESPID and Stichting Coolsingel, during the conduct of the study, which were all paid directly to a research foundation. G-J. Braunstahl reports grants from GSK, Novartis, AstraZeneca, Chiesi and Sanofi, outside the submitted work. R.W. Hendriks reports grants from Netherlands Lung Foundation, outside the submitted work. M. Kool reports grants from Netherlands Lung Foundation, during the conduct of the study.

Support statement: This study was partly supported by a long-term EAACI research fellowship to H. Vroman and a Junior investigator Grant from the Netherlands Lung Foundation (4.2.13.054JO) to M. Kool. Funding information for this article has been deposited with the Crossref Funder Registry. 


\section{References}

1 Guilliams M, Ginhoux F, Jakubzick C, et al. Dendritic cells, monocytes and macrophages: a unified nomenclature based on ontogeny. Nat Rev Immunol 2014; 14: 571-578.

2 Plantinga M, Guilliams M, Vanheerswynghels $\mathrm{M}$, et al. Conventional and monocyte-derived CD11b+ dendritic cells initiate and maintain $\mathrm{T}$ helper 2 cell-mediated immunity to house dust mite allergen. Immunity 2013; 38: 322-335.

3 Semmrich M, Plantinga M, Svensson-Frej M, et al. Directed antigen targeting in vivo identifies a role for CD103+ dendritic cells in both tolerogenic and immunogenic T-cell responses. Mucosal Immunol 2012; 5: 150-160.

4 Khare A, Krishnamoorthy N, Oriss TB, et al. Cutting edge: inhaled antigen upregulates retinaldehyde dehydrogenase in lung CD103+ but not plasmacytoid dendritic cells to induce Foxp3 de novo in CD4+ T cells and promote airway tolerance. J Immunol 2013; 191: 25-29.

5 Everts B, Tussiwand R, Dreesen L, et al. Migratory CD103+ dendritic cells suppress helminth-driven type 2 immunity through constitutive expression of IL-12. J Exp Med 2016; 213: 35-51.

6 Li JG, Du YM, Yan ZD, et al. CD80 and CD86 knockdown in dendritic cells regulates Th1/Th2 cytokine production in asthmatic mice. Exp Ther Med 2016; 11: 878-884.

7 Jenkins SJ, Perona-Wright G, Worsley AGF, et al. Dendritic cell expression of OX40 ligand acts as a costimulatory, not polarizing, signal for optimal Th2 priming and memory induction in vivo. J Immunol 2014; 179: 3515-3523.

8 Van Rijt LS, Vos N, Willart M, et al. Essential role of dendritic cell CD80/CD86 costimulation in the induction, but not reactivation, of TH2 effector responses in a mouse model of asthma. J Allergy Clin Immunol 2004; 114: 166-173.

9 Guilliams M, Dutertre CA, Scott CL, et al. Unsupervised high-dimensional analysis aligns dendritic cells across tissues and species. Immunity 2016; 45: 669-684.

10 Carr TF, Zeki AA, Kraft M. Eosinophilic and noneosinophilic asthma. Am J Respir Crit Care Med 2018; 197: 22-37.

11 Hayashi Y, Ishii Y, Hata-Suzuki M, et al. Comparative analysis of circulating dendritic cell subsets in patients with atopic diseases and sarcoidosis. Respir Res 2013; 14: 29.

12 Yerkovich ST, Roponen M, Smith ME, et al. Allergen-enhanced thrombomodulin (blood dendritic cell antigen 3, CD141) expression on dendritic cells is associated with a TH2-skewed immune response. J Allergy Clin Immunol 2009; 123: 209-216. 